\section{A rare case of acute type A aortic dissection in a patient with systemic lupus erythematosus}

\author{
Mariya Apostolova, Mahmoud Shoib, \\ Anwar Hussain \\ Conemaugh Memorial Medical Center, \\ USA
}

\begin{abstract}
Systemic lupus erythematosus (SLE) is a chronic systemic autoimmune disease. The vascular lesions in SLE usually represent small vessel vasculitis. The number of reports of vascular conditions in patients with SLE has increased possibly due to the availability of better imaging modalities and increased patient survival secondary to the use of immunosuppressive agents. However, only a limited number of cases of aortic dissection have been published in the English literature.
\end{abstract}

\section{Introduction}

We report a case of a 56-year-old African American female with a ten year history of SLE and a five year history of systemic hypertension who presented with generalized weakness, dizziness, fever, chills, cough, chest and back pain, bilateral lower and upper extremity numbness, urinary and fecal retention. The patient had been previously treated with steroids on five occasions. Each treatment lasted for close to a month for a total of 5 months in the past ten years. Computed tomography of the chest revealed the presence of a type A aortic dissection. The patient was subsequently transferred to a tertiary medical center for surgical repair. She tolerated the procedure well. Pathologic examination of the surgical specimen revealed complicated atherosclerosis with calcifications.

\section{Case Report}

A 56-year-old African American female with past medical history significant for a ten year history of SLE, five year history of hypertension and a long-standing history of cigarette smoking presented to the Emergency Department status post fall. The patient reported generalized weakness for a period of one week. Over the course of the week she became weaker with increased inability to stand and ambulate even with assistance. She had a recent history of travel by train after which she developed lower extremity swelling and pain. The above mentioned symptoms were shortly followed by the development of pleuritic chest pain. Additionally, the patient reported lightheadedness, nausea, vomiting, fever, chills, cough, chest pain, palpitations, back pain, urinary and fecal retention bilateral upper and lower extremity numbness. Home medications included aspirin, hydrochlorothiazide, lopressor, citalopram, folic acid, vitamin B, vitamin E and plaquenil.

On physical exam a high-pitch grade IVI systolic murmur was audible to auscultation over the apex of the heart. A butterfly rash was noted over her face. Tenderness to palpation was present over the thoracic and lumbosacral spine. Vitals upon admission included $\mathrm{T}$ $37.1^{\circ} \mathrm{C}$, HR 68 bpm, RR 20, BP 126/68. Cardiac enzymes, chemistries and liver enzymes were within normal limits. CBC revealed WBC 16 thou/cumm, $89 \%$ polys, Hemoglobin 13.4 gm/dL, Hematocrit 41\%,Platelets 135 thou/cumm, Sed rate $5 \mathrm{~mm} / \mathrm{hr}$.

Non-invasive studies of the lower extremities were negative for DVT. X-ray of the thoracic spine was unremarkable. Chest X-ray showed bibasilar atelectasis. X-ray of the pelvis showed mild to moderate degenerative arthritis with no evidence of acute fracture. X-ray of the lumbar spine revealed moderate degenerative spondylosis at L5-S1with no evidence of acute fracture. Computed tomography of the head showed no acute intracranial hemorrhage. Echocardiogram of the heart revealed prominent aortic root with slight intimal separation in the ascending aorta. CT of the thorax (Figure 1) revealed aortic dissection involving the ascending aorta, aortic arch and the descending thoracic aorta complicated by active intramural bleeding in its ascending portion and a discrete intimal flap in the abdominal aorta.

The patient was promptly transferred to a tertiary center for surgical repair. She underwent re-suspension of the aortic valve with creation of a neo-media and non-coronary sinus, replacement of the ascending aorta with a $28 \mathrm{~mm}$ gelweave graft, hemi-arch replacement with a beveled $28 \mathrm{~mm}$ gelweave graft. Pathologic examination of the aortic specimen revealed focally hemorrhagic adventitia, fibrous adhesions and adherent fragments of fat. The intimal surfaces of the wall showed yellow fatty streaks and plaques with occasional focal calcification. Vasculitis was not identified.

\section{Discussion}

Systemic lupus erythematosus is an autoim-
Correspondence: Mariya Apostolova, Conemaugh Memorial Medical Center, USA.

E-mail: mimoza2000bg@hotmail.com

Key words: aortic dissection, SLE, rheumatology.

Received for publication: 27 June 2011.

Accepted for publication: 12 August 2011.

This work is licensed under a Creative Commons Attribution 3.0 License (by-nc 3.0).

(C) Copyright M. Apostolova et al., 2011

Licensee PAGEPress, Italy

Rheumatology Reports 2011; 3:e6

doi:10.4081/rr.2011.e6

mune inflammatory disease characterized by the development of autoantibodies that can affect multiple organs. These multisystem disease complications can lead to severe morbidity and mortality. The cardiac manifestations of SLE include accelerated atherosclerosis, small vessel vasculitis, pericardial, valvular and myocardial disease. ${ }^{1,2}$ In a prospective study Cervera et al. ${ }^{3}$ described echocardiographic disturbances in $57 \%$ of patients diagnosed with SLE. Valvular abnormalities were detected in 44\%, pericardial effusion was identified in $27 \%$ and myocardial abnormalities were identified in $20 \%$ of the patients.

The occurrence of aortic dissection in patients with SLE is extremely rare and its pathogenesis is not clear. In a review of the published literature, Aoyagi et al. ${ }^{4}$ concluded that the majority of patients present with a long standing history of corticosteroid administration, hypertension and presence of systemic disease. These findings were supported by Wei et al. ${ }^{5}$ who reviewed 21 cases of aortic dissection occurring in SLE patients published in the current literature. The majority of the patients were young (less than 40yo) with a past medical history significant for hypertension and a long term steroid use. The average duration of SLE was 9 years. Pathologic findings were described in only 10 of the cases. Atherosclerosis was described in five, vasculitis was documented in two and degeneration in three of those cases. In the case that we described pathological examination was significant for the presence of atherosclerosis. No evidence of vasculitis was found. In contrast to the above mentioned findings, our patient was not started on a long-term corticosteroid treatment.

Several mechanisms have been suggested to explain atherosclerosis development in patients with SLE. Traditional risk factors that increase the risk of atherosclerosis, such as hyperlipidemia, hyperglycemia, hypertension, smoking, and age also increase the risk of atherosclerosis in patients with SLE. In this case, 


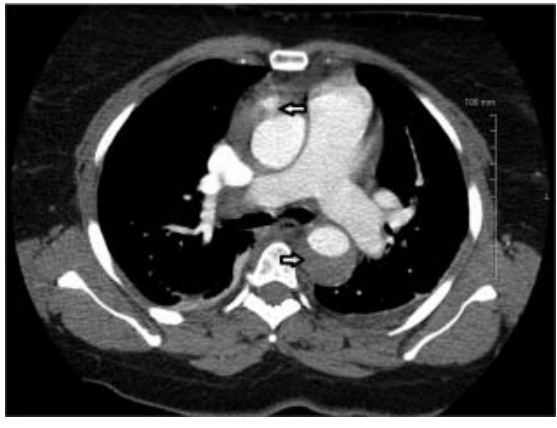

Figure 1. Computed tomography of the thorax showing aortic dissection involving the ascending aorta (top arrow), aortic arch and descending aorta (bottom arrow), complicated by active bleeding in its ascending portion (top arrow).

the patient had a long history of smoking and a five year history of hypertension. In a recent study Esdaile et $a l .{ }^{6}$ reported that SLE itself represents an independent factor in the development of premature atherosclerosis: a result of the presence of chronic inflammation leading to decreased arterial compliance and endothelial dysfunction. It has been previously hypothesized that in addition to the vasculitic inflammatory effect of SLE on the aortic wall, atherosclerosis resulting from long-term administration of steroids underlines the pathogenesis of aortic dissection in patients with Lupus. Long-term steroids were believed to promote and accelerate atherosclerosis development, increase the incidence of hypertension, and inhibit formation of chondroitin sulfate and granulation tissue which in turn leads to decrease in wall compliance and contributes to the development of aortic dissection. ${ }^{4,5,7}$ In the setting of increased shear force, as seen in hypertension, dissection could occur. However, in a recent case control study Roman et al. ${ }^{8}$ found that long term treatment with corticosteroids was not associated with an increased risk of atherosclerosis. Atherosclerosis was found to occur prematurely in patients with SLE and the condition was thought to represent an independent risk factor for cardiovascular disease. The prevalence of atherosclerosis was associated with longer disease duration and less aggressive immunosuppressive therapy. Interestingly, a significant negative correlation between the use of plaquenil and the presence of atherosclerosis was reported. In the case that we present it was speculated that the etiology of the aortic dissection was multifactorial. In addition to the chronic inflammatory effect of SLE, longterm history of smoking, the presence of systemic hypertension and atherosclerosis contributed to the development of aortic dissection.

Aortic dissection is a life-threatening condition that requires a prompt diagnosis and urgent surgical intervention. In the presence of clinical suspicion, the healthcare provider should have a low threshold for implementing imaging modalities such as ECHO and CT scan to rule out the presence of aortic dissection before the development of fatal complications. Seventy percent of patients with acute aortic dissection die within 2 weeks of onset of symptoms if not treated. ${ }^{9}$ Transesophageal echo represents an important diagnostic modality in SLE patients in renal failure. ${ }^{10}$ It has been accepted that in the general population aortic aneurysms with a diameter higher than $5 \mathrm{~cm}$ the risk of dissection increases considerably. In patients with SLE aortic dissection has been described with aortic aneurysm diameter between 3 and $6 \mathrm{~cm}$. Thus, elective surgical repair of aneurisms might be indicated earlier in SLE patients than in the general population.

\section{Conclusions}

We have described a timely ante mortem diagnosis of a rare case of aortic dissection that occurred in a patient with a history of SLE, controlled hypertension and no prior history of long-term treatment with steroids. The pathophysiology underlying the development of aortic dissection in patients with SLE is likely multifactorial. The case demonstrates that aortic dissection can occur early in the disease course even in patients who have had shortterm exposure to steroids. It further illustrates that the diagnosis of aortic aneurysm or dissection should be strongly considered in patients with prior history of SLE who present with chest or back pain. Management of traditional risk factors for atherosclerosis along with early initiation of immunosuppressive agents is advised. Since SLE represents an independent risk factor for atherosclerosis, physicians should aim at aggressive suppression of disease activity.

\section{References}

1. Kameyama K, Kuramochi S, Ueda T, et al. Takayasu's aortitis with dissection in systemic lupus erythematosus. Scand J Rheumatol 1999;28:187-8.

2. Silver AS, Shao CY, Ginzler EM. Aortitis and aortic thrombus in systemic lupus erythematosus. Lupus 2006;15:541-3.

3. Cervera R, Font J, Paré C, et al. Cardiac disease in systemic lupus erythematosus: prospective study of 70 patients. Ann Rheum Dis 1992;51:156-9.

4. Aoyagi S, Akashi H, Otsuka H, et al. Acute type A aortic dissection in a patient with systemic lupus erythematosus. Jpn Heart J 2002;43:567-71.

5. Wei HY, Chung HT, Wu CT, Huang JL. Aortic Dissection Complicated with Hemothorax in an Adolescent Patient with Systemic Lupus Erythematosus: Case Report and Review of Literature. Semin Arthritis Rheum 2010. [Epub ahead of print]

6. Esdaile JM, Abrahamowicz M, Grodzicky T, et al. Traditional Framingham risk factors fail to fully account for accelerated atherosclerosis in systemic lupus erythematosus. Arthritis Rheum 2001;44:2331-7.

7. Bulkley BH, Roberts WC. The heart in systemic lupus erythematosus and the changes induced in it by corticosteroid therapy. A study of 36 necropsy patients. Am J Med 1975;58:243-64.

8. Roman MJ, Shanker BA, Davis A, et al. Prevalence and correlates of accelerated atherosclerosis in systemic lupus erythematosus. N Engl J Med 2003;349:2399-406. Erratum in: N Engl J Med 2006;355:1746.

9. Sclair M, Nassar H, Bar-Ziv Y, Putterman C. Dissecting aortic aneurysm in systemic lupus erythematosus. Lupus 1995;4:71-4.

10. Hussain KM, Chandna H, Santhanam V. Aortic dissection in a young corticosteroidtreated patient with systemic lupus erythematosus--a case report. Angiology 1998;49: 649-52. 